\title{
Pituitary surgery is becoming more common: does this reflect a change in tumor incidence, detection, or treatment pattern?
}

\author{
Eliza B. Geer ${ }^{1}$
}

Received: 25 April 2018 / Accepted: 5 May 2018 / Published online: 16 May 2018

(c) Springer Science+Business Media, LLC, part of Springer Nature 2018

Pituitary tumors are common, comprising $10-15 \%$ of all primary intracranial tumors, and their incidence may be increasing [1]. Data from population-based cancer registries in the United States have demonstrated an annual incidence rate increase from 2.52 in 2004 to 3.13 in 2009 [2]. Other population-based studies have shown similar increases; a cohort study in Finland noted an increase in incidence from 3.8 to 4.2 cases per 100,000 population in $1992-1999$ to 2000-2007 [3]. What factors are contributing to an increase in reported rates of pituitary tumors, and how does this affect treatment patterns for these tumors?

To understand changing trends in the treatment of pituitary tumors, Crowther et al. [4] used hospital admission and Medicare data to analyze rates of hospitalizations, imaging, and surgeries in Australia between 2000/2001 and 2014/ 2015. They found that age-adjusted rates of pituitary procedures increased from 26.7 to $36.2 /$ million/year, a $35.6 \%$ increase from 2000/2001 to 2014/2015. As expected, most pituitary procedures were transsphenoidal rather than transcranial, and the proportion that were transsphenoidal increased during the period studied.

Not surprisingly, pituitary imaging, particularly MRI imaging, also increased substantially during this time. Medicare data show that MRI/CT utilization rates have almost doubled between 2000 and 2009 [5]. As noted by the authors, greater use of imaging, and improvements in diagnostic technology with increased sensitivity to identify incidental lesions, likely contributed to the increased rates of surgery seen in this study. Other studies have also shown a correlation between frequency of cranial imaging and rates of detection of incidental pituitary lesions [6].

Eliza B. Geer

geere@mskcc.org

1 Multidisciplinary Pituitary and Skull Base Tumor Center, Memorial Sloan-Kettering Cancer Center, 1275 York Ave, New York, NY 10065, USA
As the authors suggest, the observed increased rate of pituitary surgery could reflect an increase in referrals to surgery, as advances in minimally invasive surgical techniques have reduced risks associated with transsphenoidal surgery. This may or may not be related to a shift toward an endoscopic versus microscopic approach; data in Crowther et al.'s study could not differentiate a change in technique since this information was not included in the data sources used. High rates of surgery in the older age groups was also observed, in keeping with the relative safety and accessibility of this procedure.

Alternatively, the observed increase in pituitary surgery rates could reflect an actual increase in incidence of these tumors. Other studies have also suggested a higher incidence of pituitary tumors than previously noted [2]. This hypothesis is challenging to confirm, given the confounding variables, including the rise in use of imaging as mentioned. Since risk for developing a pituitary tumor increases with age, longer life expectancy may play a role as well. Finally, a higher proportion of pituitary tumors may be requiring surgery, i.e., more pituitary tumors are behaving aggressively. However, there are no data in Crowther et al.'s study to support this hypothesis, as stratification based on tumor size, invasiveness, or aggressiveness was not described.

Crowther et al. observed an increase in rates of hospital admissions for acromegaly from 2000/2001 to 2003/2004 and a subsequent decline in 2008-2009. The authors suggest this could relate to a change in IGF-1 reference ranges which lowered the diagnostic criteria for acromegaly, as well as use of a new immunoassay in Australia. A factor in the subsequent decrease in surgical rates for GH tumors could be associated with increasing use of somatostatin receptor agonists for the treatment of acromegaly.

These findings have caveats of course. Use of diagnostic codes to accurately identify cases has known limitations. In addition, the findings here do not differentiate primary versus recurrent surgeries or multiple admissions.

Limitations of database findings aside, Crowther et al. study raises interesting questions. Why is the rate of 
pituitary surgery increasing? Are pituitary tumors increasing in frequency or just being detected more frequently? Are large invasive/aggressive tumors that require surgical intervention becoming more common? Or are patients just being imaged excessively, leading to unnecessary surgery for benign, asymptomatic disease? Alternatively, are we detecting more subtle abnormalities that may benefit from intervention rather than monitoring alone, similar to the shifting diagnosis and treatment paradigms with "subclinical" or mild adrenal hypercortisolism, a diagnosis recently (and still being) defined, which developed in part as a consequence of increased rates of abdominal imaging and resulting discovery of adrenal lesions?

Answers to these questions are lacking in part due to paucity of prospective data on large cohorts of patients with pituitary tumors. Pituitary tumors are common, but pituitary tumors that are clinically significant and require surgical intervention are not common. To what extent are diagnostic imaging availability or changing assay reference ranges dictating care for pituitary patients? Large collaborative prospective studies are needed to accurately identify pituitary tumors that need intervention versus those that require surveillance only. Collection of prospective multi-center data would enhance our understanding of the natural history and treatment patterns of pituitary tumors, facilitating the development of evidence-based treatment paradigms that define appropriate intervention and follow up for patients with pituitary tumors.

\section{Compliance with ethical standards}

Conflict of interest E.B.G. has received research support for MSKCC from Novartis, Strongbridge Biopharma, and Chiasma and has served as a scientific consultant to Novartis, Strongbridge Biopharma, and Chiasma. E.B.G. declares that she has no conflict of interest.

Ethical approval This article does not contain any studies with human participants or animals performed by any of the authors.

\section{References}

1. W. Sivakumar et al. Incidental pituitary adenomas. Neurosurg. Focus 31(6), E18 (2011)

2. H. Gittleman et al. Descriptive epidemiology of pituitary tumors in the United States, 2004-2009. J. Neurosurg. 121(3), 527-535 (2014)

3. A. Raappana et al. Incidence of pituitary adenomas in Northern Finland in 1992-2007. J. Clin. Endocrinol. Metab. 95(9), 4268-4275 (2010)

4. S. Crowther et al. Trends in surgery, hospital admissions and imaging for pituitary adenomas in Australia. Endocrine 59(2), 373-382 (2018)

5. K. Lang et al. National trends in advanced outpatient diagnostic imaging utilization: an analysis of the medical expenditure panel survey, 2000-2009. BMC Med. Imaging 13, 40 (2013)

6. M.W. Vernooij et al. Incidental findings on brain MRI in the general population. N. Engl. J. Med. 357(18), 1821-1828 (2007) 